\title{
Rupturas epistémicas y complejidad en los estudios de género, una aproximación a la conciencia humana feminizada*
}

\author{
Ivonne Vizcarra Bordi ${ }^{1}$ \\ Ana Gabriela Rincón Rubio ${ }^{2}$ \\ (iD http://orcid.org/0000-0003-4456-8450 \\ Universidad Autónoma del Estado de México
}

DOI: http://dx.doi.org/10.17081/eduhum.19.33.2656

Recibido: 13 de diciembre de 2016

Aceptado: 28 de abril de 2017

\section{Epistemic ruptures and complexity in gender studies, an approximation to a feminist human conscience}

Palabras clave:

Feminismo, Estudios de género, Complejidad, Ruptura epistémica, Conciencia.

Key words:

Feminism, Gender studies, Complexity, Epistemic rupture, Conscience.

\begin{abstract}
Resumen
El objetivo de este texto consiste en reflexionar sobre los aportes feministas teóricos y empíricos que permitieron posicionamientos de rupturas epistémicas para revelar otras perspectivas de las realidades sociales y humanas. Se consideraron cinco rupturas epistémicas que desconstruyen una realidad unidimensional y racional, para dar cabida a otra compleja, que alcanzaría al espacio político de la educación transversalizada de género: biologicista, tecnocientífica, binarista, heterosexual y la separación objeto-sujeto. Se concluyó que los estudios de género pueden promover una educación des-universalizada, para dar lugar al pensamiento complejo que transciende las disciplinas y la misma conciencia humana.
\end{abstract}

\begin{abstract}
The purpose of this article consists of thinking about the theoretical and empirical feminist contributions that allowed the appearance of epistemic ruptures, thus helping to disclose the different social and human realities. Five epistemic breakdowns were hereby considered that deconstruct a one-dimensional and rational reality, facilitating the appearance of another complex real fact, the one that would reach the political educational arena of transversal gender: biologist, techno scientific, and binary, heterosexual and object-subject separation. It was concluded that gender studies, may promote a non universal education, giving space for a complex thought that transcends disciplines and human conscience itself.
\end{abstract}

\section{(c) $\$$}

Referencia de este artículo (APA): Vizcarra, I. (2017). Rupturas epistémicas y complejidad en los estudios de género, una aproximación a la conciencia humana feminizada. En Revista Educación y Humanismo, 19(33), 456-470. http://dx.doi.org/10.17081/ eduhum.19.33.2656

* Artículo de reflexión sobre rupturas epistématicas desde otras perspectivas de las realidades sociales humanas.

1. Investigadora del Instituto de Ciencias Agropecuarias y Rurales de la Universidad Autónoma del Estado de México. ivbordi@yahoo. com.mx. Línea de trabajo: Etnoconocimiento y Conciencia Ecofeminitsa.

2. Doctorante de Ciencias Agropecuarias y Recursos Naturales, Estudiante Investigadora del Instituto de Ciencias Agropecuarias y Rurales de la Universidad Autónoma del Estado de México. anarincon-mkt@hotmail.com 


\section{Introducción}

Desde finales de la década de los sesenta apareció el movimiento feminista en las universidades occidentales y occidentalizadas. Poco a poco tomó fuerza y posicionamiento académico, hasta que la puesta en marcha de programas universitarios de estudios de género y la transversalidad de la perspectiva de género, en la década de los ochenta, se convirtieron en una obligada y políticamente correcta estrategia en las agendas de educación (Ali, Shereen \& Mautner, 2004).

Las ciencias sociales, las de la conducta y las humanidades han sido eje clave de dicho posicionamiento; sin embargo, los avances teóricos propositivos para romper los paradigmas disciplinarios en esta temática han sido limitados. El mérito feminista académico es reconocido porque ha hecho posible desvelar, a través de investigaciones empíricas, las desigualdades sociales que viven las mujeres de diferentes sociedades en todas las dimensiones de la vida social y, en consecuencia, la lucha por mejorar sus condiciones se encamina hacia la formulación de políticas de igualdad. Ciertamente, estos estudios se fundamentan en diversas corrientes teóricas feministas que enriquecieron el debate científico desde una posición de ruptura (breakdown position), no solo para establecer estrategias políticas, sino para desestabilizar las teorías patriarcalizadas y racionalizadas (Barrett \& Phillips, 1992) que sujetan a las categorías subalternas, a partir de género y naturaleza, tales como sexo, raza, clase, etnia, edad, religión, recursos naturales o lo no humano
(Haraway, 1989, 1995). Michèle Barrett y Anne Phillips (1992) consideran que la crítica del conocimiento práctico por el conocimiento teórico es un proceso político, en este sentido toma su forma en las universidades cuando se politiza el conocimiento para saciar la necesidad de un adoctrinamiento racional en determinadas ideologías dominantes.

Aunque Amartya Sen (1990) empleó el concepto de posición de ruptura para explicar las desigualdades de género, los estudios feministas le han dado producción para entender los cambios sociales que provienen de quiebres abruptos en las relaciones de género fuertemente institucionalizadas como el matrimonio (Jackson, 2012), las ciencias modernas (Haraway, 1989) y el Estado (Lovenduski, 1997).

Los llamados estudios de género, con la pretensión de encontrar explicaciones convincentes sobre las desigualdades sociales, usaron al principio y con relativo éxito las mismas teorías desarrolladas en las disciplinas que emplean categorías universales (sociales, biológicas y psicológicas) (González, 2009). Sin embargo, la crítica feminista retoma estas teorías, precisamente para advertir que son miopes y carentes de verdad al no incluir la perspectiva de género (Harding \& Hintikka, 2007).

Lo anterior no significa que los estudios para denunciar las desigualdades hayan cesado, por lo contrario, ellos persisten en la tarea de la transversalización de la perspectiva de género. 
Su finalidad es incidir en el diseño y aplicación de políticas, medidas legislativas, desarrollo de programas, ejecución de proyectos u otro tipo de acciones para mejorar las condiciones de las mujeres en todos los sectores de la sociedad, en todos los niveles y etapas (Rubery, 2005) y en especial en la educación (Ali et al., 2003; Skelton, Becky \& Smulyan, 2006).

Ciertamente, esta perspectiva ha evolucionado a través de las últimas dos décadas. En concreto, ha pasado de realizar análisis sobre las relaciones de género para indagar los factores que inciden en la opresión e identificar las brechas existentes entre hombres y mujeres en cuanto al acceso y control de los recursos y a las posibilidades de desarrollo en general (Gomariz, 1992), a la necesidad de trascender las instituciones y lógicas patriarcales y racionales (Amorós, 1986). La perspectiva de género busca un mundo sin opresión, donde las diferencias biológicas formen los atributos de la igualdad, y así lograr el crecimiento personal y la conciencia de los individuos. Una vez despojados de todo tipo de prejuicios construidos en la alteridad y la otredad, porque no existe una sola condición femenina universal, tal vez estaríamos insinuando la transdisciplinariedad en términos de Nicolescu (1996). En otras palabras, los estudios de género promueven rupturas epistémicas desde la alteridad, provocando diferentes niveles de realidad; por ejemplo, el feminismo negro, el feminismo indígena, el feminismo interseccional, incluidas las nuevas masculinidades y la diversidad sexual, entre otros.
Se procura ir más allá del reconocimiento de lo establecido como realidad en un solo nivel de comprensión, aceptando, en cambio, la existencia de diferentes niveles de realidad que son regidos por lógicas disímiles. Igualmente se trata de que, desde la experiencia y saberes femeninos, se comprendan y compartan sus realidades en el entramado social que favorece el desarrollo de una conciencia humana.

La experiencia de los estudios de género en la academia y que tienen su base en las teorías feministas rupturales buscan reposicionarse en otra verdad cientifica, no dominada por ideologías patriarcales. Además, los mismas intentan promover una cultura de cambio social con igualdad, principalmente a través de todos los niveles de la educación. De esta manera, se puede decir que la construcción de un conocimiento feminista desde las mujeres conscientes ha posibilitado la aparición de un espacio político y de intervención en la enseñanza, el cual, si bien en un comienzo solo fue una pequeña resistencia contra la abismal desigualdad que existía entre los sexos, favoreció ampliamente la visibilización de los procesos políticos que se enfocaban en la parcialidad de género de la sociedad. En la actualidad, la perspectiva de género se ha posicionado en la mayoría de las agendas de políticas sociales y de educación, gracias a los centros y programas universitarios feministas y de estudios de género, que han generado una importante masa crítica que contribuye no solo a reformular estas políticas, sino a cambiar los paradigmas sobre el conocimiento universal, je- 
rárquico y androcéntrico. Estas prácticas (política y conocimiento) han evidenciado el lugar de subordinación que las mujeres han ocupado en la sociedad patriarcal como parte de un proceso histórico, desnaturalizando así: la asignación de roles y posiciones para cada sexo que la biología y la genética proponían (Schongut, 2012). Sin duda, la educación ha dado un giro enorme en el reconocimiento de la igualdad.

El presente trabajo no tiene el propósito de realizar una genealogía de los estudios de género ni de los movimientos feministas, sino recuperar de ellos los elementos teóricos y empíricos que permitieron rupturas epistémicas que revelan otras perspectivas para percibir las realidades sociales y las relaciones humanas, las cuales pueden redireccionar la construcción de nuevos modelos educativos, basados en el reconocimiento y el desarrollo de una conciencia humana y trascendental para el cambio social.

\section{Los posicionamientos de ruptura en los es-}

\section{tudios de género}

Fue necesario que pasara más de medio siglo para que la academia aceptara y validara los estudios feministas como parte del quehacer de las instituciones de educación superior.

En tal sentido, hasta la década del ochenta, las investigaciones tenían como objeto de estudio la situación de las mujeres, con énfasis en las características universales. Por un lado, sobre la división sexual del trabajo; la subordinación de la mujer en esquemas de dominación patriar- cales (familia, religión, escuela, Estado) y, por otro, de su condición femenina. Estas características fueron criticadas por otros movimientos de mujeres, especialmente afroamericanas, quienes no se sentían representadas por esas peculiaridades, pues las atravesaban otras desigualdades sociales como clase, raza, etnia, edad y generación. Inclusive se cuestionaron aspectos como las nacionalidades, profesiones y otros, imputados a los fenómenos de las desigualdades sociales entre mujeres (explotación, exclusión y discriminación), que implicaban vivencias y experiencias distintas (Barquet, 2004). Butler (2006) señala al respecto que "Nadie puede situarse en una perspectiva global del feminismo [...] que no haya sido impugnada" (p.247). Es decir, que aun con pretensiones universales, cualquier movimiento feminista tiene actitudes contestatarias y emancipadoras al igual que otros movimientos sociales con motivaciones similares como, por ejemplo, el de los pueblos indígenas en América Latina.

Los estudios feministas se concretaron a través de rupturas epistémicas importantes, donde la diversidad sociocultural y las diferencias biológicas se posicionaron en la riqueza de la igualdad social y el reclamo por la dignidad humana y la vida en general. Es en la batalla de posicionamientos de rupturas que la noción de género toma fuerza en instancias como las ciencias sociales, el psicoanálisis, la ecología y las humanidades, desde las cuales influirían directamente en el tratamiento de una política de inclusión de la perspectiva de género 
en los modelos de educación. De modo amplio, podría aceptarse que constituyen reflexiones sobre género todas aquellas que se hicieron acerca de las consecuencias y significados que tiene el pertenecer a cada uno de los sexos, donde género implica una dimensión política y la política hace al género (Scott, 1992). Así, en la década de los noventa, se consensa en el ámbito académico universitario que género es una categoría de doble propósito: analítico y político a la vez (Rubin, 1996). Analítico porque permite otra forma de conceptualizar lo femenino y lo masculino, a las relaciones entre hombres $y$ mujeres construidas social y culturalmente a partir de creencias sobre las asignaciones $\mathrm{y}$ atributos que se le otorgan a las diferencias sexuales. Político porque coloca el movimiento feminista en las tribunas jurídicas para reposicionar la lucha por la igualdad entre mujeres y hombres en todos los espacios públicos.

Ahora bien, el referente más citado y empleado para establecer una política de la perspectiva de género es el expuesto por Joan Scott (1996), para quien existe una constante en las sociedades a través de sus propios procesos históricos: todas dan como un hecho la diferencia sexual. De aquí parte la variabilidad y movilidad que pueda darse en un sistema sexo/ género "des-esencializado", cuyas relaciones de poder (estructurales e ideológicas) manifiestan desigualdades sociales entre hombres y mujeres, quienes además están adscritos a categorías sexuales diversas y con combinaciones posibles en los géneros (femenino y masculino). Martha
Lamas (1986) añade a lo anterior que las atribuciones históricas y culturales que se le asignan a cada sexo son en sí una de las variables y características que atraviesan las complejas formas de intersubjetivación que existen entre los seres humanos. Precisa también Scott (1996) que los estudios de género se alejan de la descripción de diferencias y se sitúan más bien como una categoría heurística que busca explicar, desde la hermenéutica de la sospecha, la razón por la cual la experiencia vivencial y la subjetividad que se expresa de esas experiencias, marcan las interpretaciones de la realidad y, por ende, normativizan la vida de las personas.

De este modo, se reconocen al menos cinco rupturas epistémicas que desconstruyen una realidad unidimensional y racional, para dar cabida a otra compleja, que alcanzaría al espacio político de educación transversalizada por el género:

- La biologicista y el destino de las mujeres en su papel de reproductoras de la vida humana.

- La tecnocientífica que objetiva los cuerpos sexuados para satisfacer otros placeres mercantilizados.

- Las relaciones binarias y categorías dualistas para subordinar una categoría a otra.

- La heterosexualidad como eje de la normatividad del dominio patriarcal.

- La existencia de una relación entre sujeto y objeto que anula la posibilidad de unificarlos, limitando el desarrollo de la conciencia.

\section{Desnaturalización}

Una de las primeras rupturas epistémicas 
del feminismo se basó en la desmitificación de la mujer naturalizada, basada en determinismos biológicos. Habría que mostrar al mundo que las mujeres tenían capacidades sociales similares a las de los hombres. Con la obra de Simone de Beauvoir (1999), titulada El Segundo Sexo y publicada en 1949, se cuestiona por primera vez al reduccionismo biológico haciendo célebre su frase "no se nace mujer, se llega a serlo", pues la exclusión de las mujeres del mundo de lo público realizada a través de la conceptualización de la mujer como alteridad, como naturaleza, como vida cíclica casi inconsciente, legitimó el dominio del hombre, quien se reservaba los beneficios de la civilización y del dominio patriarcal. Se trata de un reclamo cultural, construido de los estereotipos femeninos y, al mismo tiempo, un alegato en favor del reconocimiento del derecho de las mujeres, en tanto seres humanos portadores de un proyecto existencial, a acceder al mundo de la cultura del que políticamente se les excluyó. Los feminismos liberal, socialista y radical de principios de los años setenta del siglo $\mathrm{XX}$ han recogido esta reivindicación, consiguiendo romper en gran parte una de las primeras ataduras del orden social basado en la división sexual del trabajo, donde por la asignación de los roles de género, a las mujeres les corresponde la esfera doméstica y de la reproducción biológica y social (Scott, 1996).

Algunas feministas anglosajonas dedicaron sus estudios a mostrar cómo ciertos mecanismos institucionalizados, no solo por el sistema de creencias sino por las ciencias, las artes y las tecnologías, legitimaban dicho dominio ( $\mathrm{u}$ orden). Una de las mayores referentes que impulsó el feminismo radical fue Betty Friedan (2009), quien con su obra La mística de la femineidad (la imagen de lo esencialmente femenino) publicada en 1963, analizó el rol social femenino construido a través de las revistas, la publicidad y la literatura, que condicionaba la vida y felicidad de muchas mujeres estadounidenses expulsadas del mercado de trabajo de la postguerra. Esta ha sido una obra influyente y decisiva en el acompañamiento de uno de los cambios sociales más determinantes del siglo XX: la posición y autoconciencia de las mujeres de sus cuerpos y como grupo.

Ciertamente estas obras aisladas no tuvieron repercusión en su momento, pero fueron retomadas posteriormente por el feminismo académico universitario. La Organización Mundial para las Mujeres en Estados Unidos de América, fundada por Friedan en 1966, agrupó a un número importante de académicas y activistas estadounidenses, llamadas feministas liberales de la segunda ola (1960-1990), quienes forjaron una política para impulsar programas universitarios que atendieran las problemáticas femeninas, y abrirles de esta manera espacios a las mujeres en el sector público para lograr la igualdad social (Perona, 1994). Si bien la primera ola del feminismo (1840-1950) se caracterizó principalmente por movimiento sufragista con tintes de igualdad en el reconocimiento al derecho, no fue hasta la segunda ola, donde la agenda de la igualdad tuvo mayor impacto en el 
ámbito público, gracias a la influencia feminista en los esquemas académicos que sirvieron de plataforma para la mayoría de los programas $\mathrm{y}$ centros en todo el mundo universitario occidental incluyendo los países latinoamericanos (Barquet, 2004). La producción científica feminista diversificada produjo varias corrientes del pensamiento feminista, dando lugar en la década de los noventa, al impulso de la tercera ola, y con ella, se han expandido las políticas de igualdad, con la cual se constata el impacto de la internacionalización y la globalización de los movimientos feministas (Valcárcel, 2012).

\section{Desobjetivación del cuerpo sexuado}

Uno de los aportes más significativos de las feministas de la segunda ola consistió en adueñarse del cuerpo, la sexualidad y la salud reproductiva, con lo que se anuncia la segunda ruptura epistémica. Por un lado, "los estudios sobre la sexualidad estaban fuertemente implicados en una visión neutral del desarrollo de la ciencia, donde el conocimiento científico impregnado en los textos de enseñanza de educación básica y media superior pretendía ser universal e imparcial, que junto a una noción de objetividad absoluta como guía de la ciencia tradicional, generaban una dicotomía entre los hechos (científicos, objetivos, imparciales) y los valores" (Schongut, 2012, p.37). Por otro lado, algunos estudios feministas denunciaron la fragmentación del cuerpo y la sexualidad de las mujeres porque limitaban su pleno conocimiento, y por lo tanto impedían la liberación sexual al sujetar a los cuerpos sexuados a un tratamiento de objetivación, separado de las subjetividades (Butler, 2002). Así, la objetivación del cuerpo ha sido reconocida como territorio político, dado que los aspectos más fundamentales de la existencia corporal de las mujeres constituían elementos significativos en la construcción social de normas opresivas para ellas (Vizcarra \& Rincón, 2015).

En la ruptura de la opresión surge el cuerpo que "apelará la experiencia, a la corporalidad. La que hace referencia a la realidad subjetiva, vivenciada o experienciada; por ello está en la intencionalidad de la vida psíquica, mental y espiritual. El cuerpo no objeto ni diluido ni separado en la abstracción, es la expresión de la corporalidad; es la historia vital interna, madura hacia la diferenciación; en tanto cada cual tiene su propia historia individual y no se limita al volumen del cuerpo, sino que es capaz de extenderse e incluso tomar posesión de los objetos simbólica y materialmente (Montenegro et al., 2006, citado en Vizcarra \& Rincón, 2015, p. 64).

La crítica feminista al modelo tradicional de hacer ciencia (objetivo, a-ideológico, a-político) y tecnología (mercantilización del conocimiento) sería fundamental para dar cuenta de los sesgos de género con que el modelo ya venía impregnado, tanto en sus formas como en su contenido. Para Rodríguez (1999), el capitalismo patriarcalizado pretende diluir lo real y considerar el mundo como pura abstracción sin anclaje real, por lo que el problema reside en determinar hasta qué punto lo real, el cuerpo 
vivido (viviente), ha devenido para la educación, las ciencias y las tecnologías, en un cuerpo abstracto, ilusorio e impalpable, un cuerpo sin rostro, sin experiencia y, por lo tanto, sin vida.

\section{Antibinarismos}

Paralelamente a este debate crítico de la tecnociencia, se despliega otro rompimiento epistémico hacia finales de los setenta, relacionado ahora con las posiciones binarias y dualistas, que conciben al mundo en relaciones asimétricas y jerarquizadas, fortaleciéndose con ello las sociedades patriarcales e industrialistas basadas en el desarrollo de las ciencias modernas: Naturaleza/Cultura, Mujer/Hombre, Privado/Público, Cuerpo/Mente, Afectividad/ Racionalidad, Espíritu/Materia (Plumwood, 1991). Para des-esencializar estas relaciones binarias y superar los dualismos jerarquizados, se requirió de estudios filosóficos y se transitó hacia el constructivismo, a la sociocrítica, a la ecología política, al postmodernismo y a los estudios poscoloniales, todos los cuales propiciaron el desarrollo de varias corrientes teóricas llamadas, por ejemplo, ecofeministas, ecología feminista, ambientalistas femeninas o ecología política feminista (Vizcarra \& Rincón, 2015).

Estas corrientes coinciden por lo general en que la Naturaleza no solo se refiere a los fenómenos que ocurren para que los seres vivientes interactúen en ambientes y sistemas complejos, sino a la comprensión de lo que significa vida, de vivir y pensar en su totalidad el sentido de la humanidad (Morin, 1995). Alicia
Puleo (2011) concluye que la conciencia humana ha de avanzar hacia la igualdad de mujeres y hombres, en tanto partícipes no solo de la cultura sino también de la naturaleza, revalorando su sentido de relacionar la vida humana y no humana. Esto incluye tanto el reconocimiento de las mujeres en el ámbito de la cultura como la plena aceptación, en lo propiamente humano, de elementos despreciados y marginalizados como femeninos (los lazos afectivos, la compasión, la materia, la naturaleza).

Esta última dimensión trajo a su vez nuevas corrientes feministas, unas más críticas que otras. Por ejemplo, el llamado "feminismo de la diferencia", propuesto por Luce Irigaray a través de su tesis doctoral titulada Spéculo de la otra mujer (1978). Sostiene que es importante construir la feminidad a partir de una teoría basada en la diferencia sexual con valores propios, por lo que se requiere desestabilizar el ego masculino androcéntrico fálico. Esta polémica provocó su expulsión de la Universidad de Paris 8 Vincennes-Saint-Denis.

A partir de esta última experiencia, Irigaray se desentiende de la pro-igualdad como estandarte feminista y defiende su postura por el reclamo de una "subjetividad femenina autónoma", aduciendo que en la historia, el lenguaje, la psicología y todas las disciplinas, lo femenino se ha definido a partir de lo masculino y necesita, por tanto, un reconocimiento propio desde la esfera política. De hecho, ella denunció que para posicionar el feminismo en las universidades, 
hay que convencer políticamente a quienes dirigen el conocimiento científico y humanístico.

\section{Diversidades}

Paralelamente a estas posturas, surge la obra de Judith Buttler (2001), la cual permite acercarnos a las rupturas epistémicas más recientes que trascienden la academia. Pone en entredicho la heterosexualidad como norma exclusiva de la zona de ser "normal", desarrollando la 'Teoría Queer', para afirmar que la orientación sexual y la identidad sexual o de género de las personas es resultado de una construcción social y que, por tanto, no existen papeles sexuales esenciales o biológicamente inscritos en la naturaleza humana, sino formas socialmente variables de desempeñar uno o varios papeles sexuales. Buttler ha modernizado el feminismo adaptándolo a las diversidades sexuales, que incluye a las personas transexuales y transgéneros y que no se sienten definidas por los sustantivos "mujer" o "hombre" o por lo "femenino" y "masculino". Su intención fue debatir acerca de los planteamientos existentes hasta la fecha sobre el género atado a la heterosexualidad, que limitaban su significado a los conceptos sistémicos y generalmente aceptados de: hombre-masculinidad y mujer-feminidad (González, 2009). En la actualidad, se reconoce que las contradicciones internas y rupturas históricas asociadas a la feminidad y a la masculinidad y sus diversidades sexuales, forman parte de los estudios de género y conforman el conjunto de argumentos políticos empleados para reivindicar los derechos de los movimientos gay, lésbico, transexuales, trans e intergéneros.
De la misma manera, las masculinidades y nuevas masculinidades se integraron al entramado de estos estudios gracias a las investigaciones de Robert W. Connell (1995), con su trabajo Masculinities. Para el autor, la(s) masculinidad(es), así como la(s) femineidad(es) se asocian a configuraciones conductistas limitadas por la "personalidad" o el "carácter". Según Moya et al. (2001), durante mucho tiempo se consideró erróneamente a la masculinidad y a la feminidad como una dimensión de dos polos de comportamiento psicosocial, con el propósito de clasificar a las personas en uno específico: mujer/femenino y hombre/ masculino, pero nunca ambos sistemas a la vez ni todas sus combinaciones posibles. Asimismo, los roles sexuales estaban rígidamente ligados al sexo biológico, de manera que el ser masculino o femenino dependía básicamente de ser hombre o mujer. Sin embargo, esta concepción empezó a ser cuestionada, surgiendo en la década de los setenta una nueva concepción de la masculinidad y feminidad como dos dimensiones independientes, de tal forma que las personas obtienen puntuación por separado en cada una de ellas. Desde esta perspectiva, hombres y mujeres son mucho más parecidos en su psicología de lo que tradicionalmente se asumía, aunque Connell (1995) admite el dominio masculino como referente de lo femenino. De aquí se refuerza la necesidad de deconstruir la versión de la realidad forjada por la identidad humana masculinizada, puesto que, "la manifestación más evidente y más extrema de la masculinización de nuestro mundo es la aparición a todos los niveles 
de la vida social del culto de la personalidad" (Nicolescu, 1996, p.77). Con ello se aleja del esencialismo que tanto critican los estudios de género, y establece una nueva línea de debate dentro de estos, pues los hombres tampoco son objetos sexuados, sino sujetos dominados por un sistema de creencias contextuales y patriarcales.

Para avanzar en materia de igualdad en la diversidad desde las bases de educación, habría que implementar políticas que tengan como objetivo promover no solo una igualdad efectiva y real para las mujeres y los hombres en la edad pre-escolar, sino erradicar los roles de cada uno en función del mantenimiento de una sociedad tradicionalmente sexista (Castillo-Bolaños \& Morales-Ortega, 2013) y que se reproduce en los espacios escolares.

\section{La relación objeto-sujeto}

Por su extensa producción de conocimientos, se puede decir que los estudios de género que se han ocupado de la experiencia humana, se enfrentan a una ruptura epistémica compleja, es decir, la que no separa al sujeto del objeto. Necesariamente esta tarea debe tener al menos tres consideraciones en el diseño de nuevas propuestas de educación con perspectiva de género:

Primera: debido a su relación con la alteridad, con la otredad y con el poder ejercido de uno-a sobre otra-o, siempre se debe partir de un contexto relacional que incursione tanto en la subjetividad como en la intersubjetividad (Burin \& Meler, 2000).
Segunda: alejarse de una visión esencialista, centrada en determinar las características y diferencias individuales. Ello provee de herramientas para acercarse a los enfoques psicosocial y socio-cultural, que proponen la reproducción de los estereotipos de género aprendidos y transmitidos por generaciones a través de procesos de convivencia social que no solo adscriben características y roles diferenciados a hombres y mujeres, sino que definen sus esquemas de referencia, influyendo así en sus expectativas y comportamientos y reforzando, en última instancia, la continuidad de la segregación formativa y ocupacional (Sagebiel \& Vázquez, 2010).

Tercera: El género no es una adscripción de moda a una política ni una noción totalizadora, pues envuelve otras variables que entrecruzan aspectos igualmente relevantes de la subjetividad humana, tales como clase, etnia o raza, edad y religión (Burin \& Meler, 2000). Por ello, la categoría género no subordina a ninguna otra categoría ni pretende subordinar la misma que la ha dominado, sino que se libera de la sujeción (Vizcarra \& Rincón, 2015).

\section{Conciencia feminista-humanista}

Enseñar a partir de una ética feminista es persuadir al humano a comprender la diversidad de los seres como individuo-sociedad-especie, de manera conjunta y simultánea. Esta ética ha sido construida en la experiencia de los estudios de género en la educación superior en busca de un paradigma fundado en la concientización de 
la vida humana y no humana, libre de violencia. Es decir, que sabiendo cómo los feminismos han transgredido las disciplinas a través de sus posiciones de rupturas epistémicas del conocimiento, podemos proponer modelos educativos que retomen la complejidad del pensamiento humano a través de la mirada transdisciplinar (entre las disciplinas, a través de las disciplinas y más allá de cualquier disciplina). La finalidad es enseñar a comprender el mundo presente de las relaciones de género, tanto como especie humana en la unidad frente a la diversidad (etnia, clase, religión, costumbres familiares), como en las dimensiones conocidas (ontológicas, empíricas, axiológicas, epistemológicas y metodológicas) y desconocidas (innombrables por ello) en las que se relacionan.

El ejercicio de las rupturas epistémicas que ofrecen los estudios de género para percibir y relacionarnos con otros niveles de realidades no dominantes, permite reflexionar en la necesidad de construir un modelo de educación alternativo. Un modelo en el que docentes y estudiantes se impliquen en una actitud de apertura con la vida y de todos sus procesos (Moraes, 2008). Ello daría lugar a procesos de aprendizajes de otras realidades y formarían un tipo de masa crítica para elevar la conciencia que trate de comprender lo que está más allá del orden social establecido. En otras palabras, la experiencia de los estudios de género nos ha enseñado a ejercitar los aprendizajes de la reconexión, de la complejidad y del amor, lo que implica también la necesidad de trabajar con la multidimensionalidad humana desde estrategias de aprendizaje que envuelvan no solo los aspectos racionales, técnicos $\mathrm{y}$ simbólicos, sino también los aspectos intuitivos, míticos, mágicos y afectivos, así como los múltiples idiomas que dan sentido y significado a la existencia humana (Moraes, 2008).

\section{Consideraciones finales}

El profesorado que se adscribe a los estudios de género en educación superior está aportando y construyendo una comunidad de rupturas epistémicas, para dar paso a otras formas de relacionarnos.

Si bien para González (2009) no existe tal construcción y, al asumirlo, se corre el riesgo de permanecer como un grupo cerrado y sin influencia, para que con rigor feminista se argumente la importancia de la transversalidad de la perspectiva de género en los planes de educación en todos los niveles, estamos a tiempo de advertir los cambios que se quieren para construir conciencia de otras realidades de paz, armonía, justicia y afectividades.

En efecto, por un lado, para trascender los estudios de género, estos deben tener una acepción más relacionada con la afectividad que con la efectividad del precepto mismo. Esto porque la afectividad está adscrita a lo femenino, a la feminidad de los seres humanos y por ello no tiene valor mercantil: por eso es ridiculizada, ignorada, olvidada y aún menospreciada por el sistema dominante, que disfraza la inclusión con 
políticas de igualdad sin despatriarcalizar los mismos sistemas (Nicolescu, 1996).

Por otro lado, los estudios de género que en un principio buscaban la liberación del yugo patriarcal, poco a poco trascendieron hacia la búsqueda de la igualdad y han favorecido el ejercicio de una lectura crítica y cuestionadora de la realidad mercantilizada, dirigida a analizar y transformar la situación de las personas. En la actualidad, nos encontramos en un momento de crear nuevas construcciones con otras realidades humanas y con diferentes niveles de percepción, a través de vínculos afectivos no jerarquizados ni discriminatorios, y que procuran poner distancia de las políticas de igualdad no despatriarcalizadas. Nicolescu (1996) advierte al respecto que guardar una orientación constante en el atravesamiento de estos niveles garantiza una afectividad creciente que asegura la unión entre todas las personas del planeta; entre nosotros y nosotras y los seres vivos no humanos.

Los estudios de género han puesto en la mira la complejidad de la educación. La reflexión conlleva a distanciarnos (aunque sea de manera imperfecta y temporal) de los hechos clave de nuestro sexo, nuestra religión, nuestra nacionalidad, nuestra clase, nuestras creencias, para participar imaginativamente en experiencias muy diferentes de las nuestras. Ello no significa que tengamos que negar estos rasgos; pero, por ahora, como lo central en un contexto es marginal en otro, tal ejercicio es lo que nos permite cambiar de perspectiva sobre la condición humana y colocar la ética del género humano como uno de los saberes de la educación del futuro que propuso Edgar Morin (2013).

Es probable que con esta conciencia femenina que proviene de la lucha por derrocar la ideología patriarcal en todos los ámbitos de la vida humana, empiece lo que Nicolescu (1996) llama "muerte del hombre", en términos de una etapa de la historia. Ahí se augura un segundo nacimiento, el de la humanidad des-universalizada, sin pretensiones de sujetar a los objetos. Es decir, una humanidad despatriarcalizada, sin masculinidad ni feminidad, donde no haya cabida al mercantilismo y donde la tecno-ciencia sirva al desarrollo de la conciencia y tienda, por lo tanto, a abolir la jerarquización y la opresión.

En su manifiesto, Nicolescu (1996) presume que la feminización social ha posibilitado la apertura de otros niveles de percepción. Generar nuevos conocimientos de estas otras realidades a partir de la experiencia feminista en los estudios de género, posiciona entonces a la feminidad, con su amplio reconocimiento de la diversidad, como una fortaleza. Sin duda, ello cambiará la educación convencional universalizada-antropocéntrica, pero una vez conquistado el segundo nacimiento, este evolucionará en la comunión de la humanidad y la naturaleza. Ciertamente, para que eso suceda, en definitiva la propuesta de la transdisciplinariedad en la educación con perspectiva de género es una vía más segura que el de la confrontación en las tribunas científicas y políticas aún masculinizadas. 


\section{Referencias}

Ali, S., Shereen, B. \& Mautner, M. (Eds.) (2004). The Politics of Gender and Education. Critical Perspectives. Nueva York: Palgrave, Macmillan.

Amorós, C. (1986). Hacia una crítica de la razón-patriarcal. Madrid: Anthropos.

Barquet, M. (2004). Teorías de género, breve actualización. Contexturas, 5 ( 13 ) (separata).

Barrett, M. \& Phillips, A. (Comps.) (1992). Desestabilizar la teoría. Debates feministas contemporáneos. México: Tribunas políticas. UNAM/Paidós.

Burin, M. \& Meler, I. (2000). Género. Una herramienta teórica para el estudio de la subjetividad masculina. En Burin, M. \& Meler I. (eds.). Varones: Género y subjetividad masculina. (pp.21-70) Argentina: Paidós.

Butler, J. (2001). El género en disputa. El feminismo y la subversión de la identidad. México: Paidós.

Butler, (2002). Cuerpos que importan. Sobre los límites materiales y discursivos del sexo. Buenos Aires: Paidós.

Butler, (2006). Deshacer el género. Barcelona: Paidós.

Castillo-Bolaños, J. \& Morales-Ortega, H. (2013). Los estudios de género a las nuevas masculinidades y/o los movimientos de padres por la custodia compartida de sus hijos e hijas. Educación y Humanismo, 15(24),107-121. Disponible en: http:// portal.unisimonbolivar.edu.co:82/ rdigital/educacion/index.php/educacion . Consultado el 19 de noviembre de 2016.
Connell, R. (1995). Masculinities. Cambridge: Polity Press.

De Beauvoir, S. (1999). El segundo sexo. Buenos Aires: Siglo XX.

Friedan, B. (2009). La mística de la feminidad. Madrid: Cátedra, Colección Feminismos.

Gomariz, E. (1992). Los estudios de género y sus fuentes epistemológicas. Periodización y perspectivas. ISIS Internacional, Ediciones de las Mujeres, (17), 1-48.

González, R. (2009). Estudios de género en educación: una rápida mirada. Revista mexicana de investigación educativa, 14(42), 681-699.

Haraway, D. (1989). Primate Visions: Gender, Race, and Nature in the World of Modern Science. Londres: Routledge.

Haraway, D. (1995). Ciencia, cyborgs y mujeres. La reinvención de la naturaleza. Madrid: Cátedra.

Harding, S. \& Hintikka, M. (eds.) (2007). Discovering reality: feminist perspectives on epistemology, metaphysics, methodology and philosophy of science. Dordrecht, Los Países Bajos: Kluwer Academic Publishers.

Irigaray, L. (1978). Spéculo de la otra mujer. Madrid: Saltés.

Jackson, C. (2012). Marriage, Gender Relations and Social Change. Journal of Development Studies, Routledge, part of the Taylor \& Francis Group, 48(1), 1-9.

Lamas, M. (1986). La antropología feminista y la categoría "género". Nueva Antropología, VII(30), 173-198.

Lovenduski, J. (1997). Feminismo institucional, 
género y Estado. En E. Uriarte \& E. Aramtxa (Coords.). Mujeres en política: análisis y práctica (pp. 201-210). España: Ariel.

Montenegro, M. A., Ornstein, C. \& Tapia, P.A. (2006). Cuerpo y corporalidad desde el vivenciar femenino. Acta Bioéthica, OPS, XII, 165-168.

Moraes, M. (2008). Ecologia dos Saberes: Complexidade, transdisciplinaridade e educação. São Paulo: Antakarana/ PróLibera.

Morin, E. (1995). Introducción al pensamiento complejo. España: Editorial Gedisa.

Morin, E. (2013). Los siete saberes necesarios para la educación del futuro. México, Dower International.

Moya, M., Páez, D., Glick, P., FernándezSedano, I. \& Poeschl, G. (2001). Sexismo, masculinidad-feminidad y factores culturales. Revista Electrónica de Motivación y Emoción, 4 (8-9). Disponible en línea: http://reme.uji.es/articulos/ amoyam4101701102/texto.html.

Nicolescu, B. (1996). La transdisciplinariedad, Manifiesto. París: Ediciones $\mathrm{Du}$ Rocher. Recuperado en: http:// es.scribd.com/doc/38437874/BassarabNicolescu-La-TransdisciplinariedadManifiesto1\#scribd.

Perona, A. (1994). El feminismo americano de post-guerra, B. Friedan. En C. Amorós (Coord.). Actas del seminario Historia de la teoría feminista. Madrid: Instituto de Investigaciones Feministas, Universidad
Complutense de Madrid.

Plumwood, V. (1991). Nature, self, and gender: Feminism, environmental philosophy, and the critique of rationalism, En K. Warren, y C. Macalester. Hypatia, Special Issue; Ecological Feminism. 6, 3-27.

Puleo, A. (2011). Ecofeminismo, para otro mundo posible. Madrid: Ediciones Cátedra.

Rodríguez, R. (1999). Tras los recorridos de las nociones de corporalidad y experiencia desde una perspectiva feminista. Recuperado el 20 de marzo de 2014 en: www. publicaciones.ffyh.unc.edu.ar/ index.php/6encuentro/article/.../71/65.

Rubery, J. (2005). Reflections on gender mainstreaming: an example of feminist economics in action? Feminist Economics, $11(3), 1-26$.

Rubin, G. (1996). El tráfico de mujeres: notas sobre la 'economía política del sexo'. En M. Lamas (Comp.). El género: la construcción cultural de la diferencia sexual (p.35). México: Porrúa/ Programa Universitario de Estudios de GéneroUNAM.

Sagebiel, F. \& Vázquez Cupeiro, S. (2010). Topic Report. Meta-analsis of gender and Science Research. Stereotypes and Identity. Disponible en: http://genderedinnovations.stanford.edu/images/TR3 Stereotypes.pdf. Consultado el 23 de noviembre de 2016.

Scott, J. (1992). Igualdad versus diferencia: los usos de la teoría posestructuralista. Debate Feminista, 3(5), 85-104.

Scott, J. (1996). El género: una categoría útil 
para el análisis histórico. En M. Lamas. (Comp.), El género: la construcción cultural de la diferencia sexual (pp.265302). México: Porrúa/Programa Universitario de Estudios de Género-UNAM.

Schongut, N. (2012). La construcción social de la masculinidad: poder, hegemonía y violencia. Psicología, Conocimiento y Sociedad, 2(2), 27-65. Revisiones.

Sen, A. (1990). Gender and Cooperative Conflicts. In I. Tinker (ed.), Persistent Inequalities. Women and World Development (pp.1-58). Oxford: University Press Oxford.

Skelton, C., Becky, F. \& Smulyan, L. (Eds.)
(2006). The Sage Handbook of Gender and Education. Londres: SAGE Publications.

Valcárcel, A. (2012). Feminismo en un mundo global. Madrid: Cátedra.

Vizcarra, I. \& Rincón, A. (2015). Cuerpo, espíritu y naturaleza en los estudios de género y ambiente. En E. Zapata \& M.R. Ayala (Coords.), Contribuciones de los estudios de género al desarrollo rural (pp.63-88). México: COLPOS. 